\title{
\begin{tabular}{l|l} 
MitTraries & DSpace@MIT
\end{tabular}
}

\author{
MIT Open Access Articles
}

\section{A procedure for testing the significance of orbital tuning of the martian polar layered deposits}

The MIT Faculty has made this article openly available. Please share how this access benefits you. Your story matters.

Citation: Sori, Michael M. et al. “A Procedure for Testing the Significance of Orbital Tuning of the Martian Polar Layered Deposits." Icarus 235 (2014): 136-146.

As Published: http://dx.doi.org/10.1016/j.icarus.2014.03.009

Publisher: Elsevier

Persistent URL: http://hdl.handle.net/1721.1/110320

Version: Author's final manuscript: final author's manuscript post peer review, without publisher's formatting or copy editing

Terms of use: Creative Commons Attribution-NonCommercial-NoDerivs License 


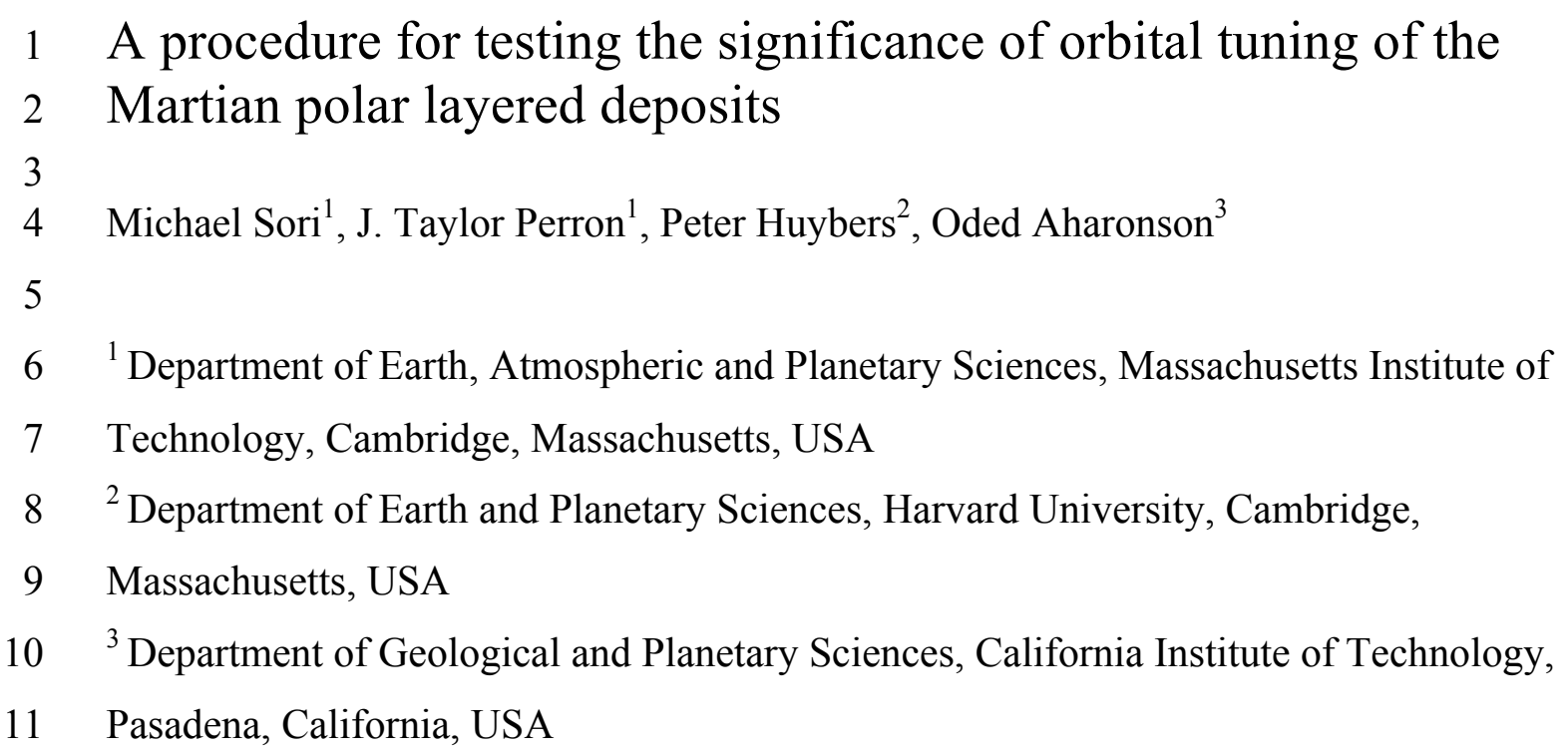

\section{ABSTRACT:}

16 Layered deposits of dusty ice in the Martian polar caps have been hypothesized to

17 record climate changes driven by orbitally induced variations in the distribution of

18 incoming solar radiation. Attempts to identify such an orbital signal by tuning a

19 stratigraphic sequence of polar layered deposits (PLDs) to match an assumed forcing

20 introduce a risk of identifying spurious matches between unrelated records. We present

21 an approach for evaluating the significance of matches obtained by orbital tuning, and

22 investigate the utility of this approach for identifying orbital signals in the Mars PLDs.

23 Using a set of simple models for ice and dust accumulation driven by insolation, we

24 generate synthetic PLD stratigraphic sequences with nonlinear time-depth relationships.

25 We then use a dynamic time warping algorithm to attempt to identify an orbital signal in

26 the modeled sequences, and apply a Monte Carlo procedure to determine whether this

27 match is significantly better than a match to a random sequence that contains no orbital 
28 signal. For simple deposition mechanisms in which dust deposition rate is constant and

29 ice deposition rate varies linearly with insolation, we find that an orbital signal can be

30 confidently identified if at least $10 \%$ of the accumulation time interval is preserved as

31 strata. Addition of noise to our models raises this minimum preservation requirement, and

32 we expect that more complex deposition functions would have a similar effect. In light of

33 these results, we consider the prospects for identifying an orbital signal in the actual PLD

34 stratigraphy, and conclude that this is feasible even with a strongly nonlinear relationship

35 between stratigraphic depth and time, provided that a sufficient fraction of time is

36 preserved in the record and that ice and dust deposition rates vary predictably with

37 insolation.

\section{1. Introduction}

40 The topographic domes of the north and south polar ice caps on Mars are mostly

41 composed of kilometers-thick layered sedimentary deposits, the polar layered deposits

42 (PLDs), which are exposed in spiraling troughs cut into the caps [Murray et al., 1972;

43 Cutts, 1973; Howard et al., 1982; Byrne, 2009], as shown in Figure 1. The PLDs were

44 initially seen in images from the Mariner 9 spacecraft [Murray et al., 1972], and were

45 immediately inferred to be composed of atmospherically deposited dust and ice [Cutts,

46 1973]. Since then, the PLDs have been more thoroughly characterized. Carbon dioxide

47 ice and clathrate hydrate have been shown to be compositionally insignificant based on

48 their effects on thermal properties [Mellon, 1996] and bulk strength [Nye et al., 2000].

49 Water ice dominates dust volumetrically; dust volume composition has an upper limit of

$502 \%$ in the north polar cap [Picardi et al., 2005] and 10\% in the south polar cap [Plaut et 
51 al., 2007] according to MARSIS radar transparency data, and $~ 15 \%$ in the south polar

52 cap according to gravity anomalies associated with the area [Zuber et al., 2007;

53 Wieczorek et al., 2008]. Concentrations far smaller than these upper bounds could

54 produce the observed brightness differences [Cutts, 1973]. MOLA topography

55 demonstrates that the ice caps are dome-like structures 3-4 km thick [Zuber et al., 1998],

56 with volumes of 1.14 million $\mathrm{km}^{3}$ for the northern dome [Smith et al., 2001] and 1.6

57 million $\mathrm{km}^{3}$ for the southern dome [Plaut et al., 2007]. The deposits are locally overlain

58 by seasonal carbon dioxide frost [Smith et al., 2001]. Radar soundings from the

59 SHARAD instrument [Phillips et al., 2008] have revealed that large-scale stratigraphy is

60 similar in different parts of the northern ice cap, implying that the PLDs record regional

61 or global climate phenomena rather than local conditions.

62 Many authors have attempted to constrain the deposition rates of polar ice or dust

63 [Pollack et al., 1979; Kieffer, 1990; Herkenhoff and Plaut, 2000], but these estimates

64 span orders of magnitude. Populations of impact craters on the polar caps provide some

65 constraints, including an estimated mean surface age of 30 to $100 \mathrm{Myr}$ for the southern

66 PLDs [Koutnik et al., 2002] and an estimated upper limit on the accumulation rate of 3-4

$67 \mathrm{~mm} / \mathrm{yr}$ for the northern PLDs [Banks et al., 2010]. Despite these efforts, the ages of the

68 PLDs remain poorly constrained.

69 It has been proposed that patterns in the thickness and brightness of these layers,

70 which are thought to result from variable dust concentration in the ice, are controlled by

71 changes in the distribution of solar radiation due to quasi-periodic variations in the

72 planet's spin and orbital characteristics over time, specifically climatic precession,

73 obliquity variation, and eccentricity variation [Murray et al., 1973; Cutts et al., 1976; 


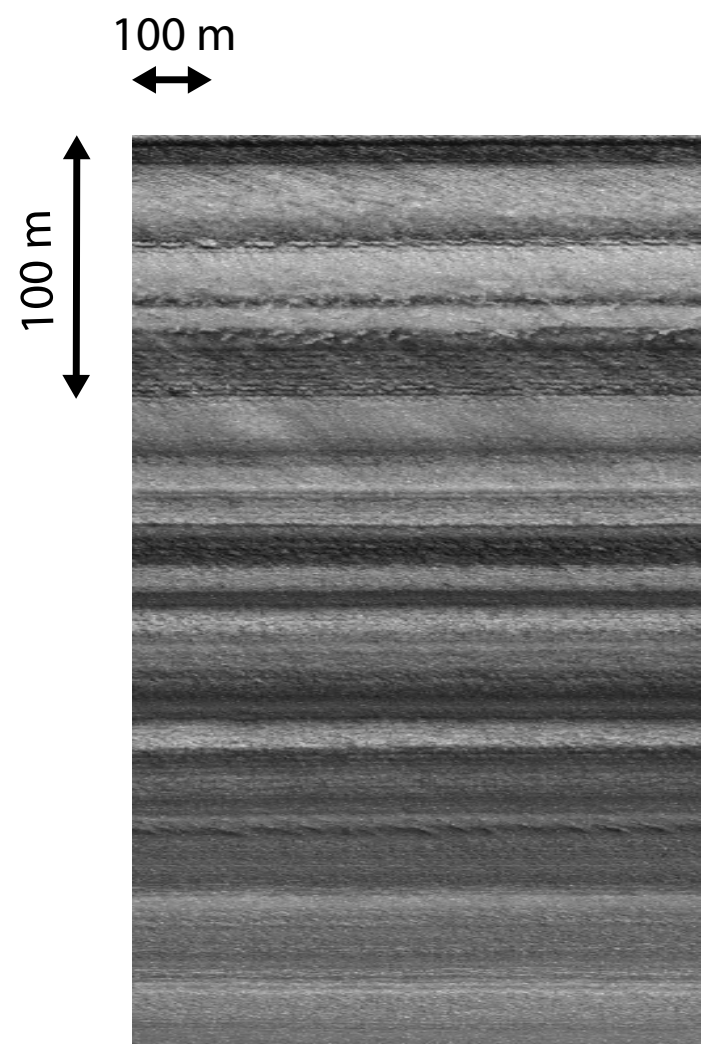

Figure 1. Mars Orbiter Camera (MOC) Image \#M0001754 of a PLD stratigraphic sequence, corrected for topography. The vertical scale corresponds to vertical depth within the PLD sequence, and the horizontal scale corresponds to distance along the outcrop.

74 Toon et al., 1980; Cutts and Lewis, 1982; Howard et al., 1982; Thomas et al., 1992,

75 Laskar et al., 2002; Milkovich and Head, 2005; Milkovich et al., 2008; Fishbaugh et al.,

76 2010]. In this way, the PLDs may record past Martian climate.

77 An analogous argument is often made regarding ice cores or marine sediment

78 cores and Earth's paleoclimate. Some of the variability in marine Pleistocene

79 paleoclimate proxies has been convincingly linked to orbital changes [Hays et al., 1976].

80 However, there is debate about how much of the recorded climate variability was

81 deterministically controlled by Milankovitch cycles [Kominz and Pisias, 1979; Wunsch,

82 2004]. In theory, the problem on Mars should be more tractable than the analogous

83 problem on Earth. The Martian atmosphere is orders of magnitude less massive than 
84 Earth's, and Mars has not had a surface ocean in the recent past, two factors that should

85 make the Martian climate system simpler than the terrestrial one. Mars also experiences

86 larger obliquity and eccentricity variations than Earth [Ward, 1973; Touma and Wisdom,

87 1993; Laskar et al., 2004], which should make an orbital signal, if present, stronger and

88 perhaps easier to detect.

89 Despite the likelihood of a simpler climate on Mars, detection of an orbital signal

90 in the PLDs is not a trivial task. The relationship between time and stratigraphic depth in

91 the PLDs is unknown, and is likely nonlinear. There are no absolute ages available for

92 any part of the deposits. Image brightness may contain noise from image artifacts,

93 inherent noise in the deposition rates of ice and/or dust, and an indirect relationship

94 between visible albedo and PLD composition [Tanaka, 2005; Fishbaugh and Hvidberg,

95 2006; Herkenhoff et al., 2007; Levrard et al., 2007]. Because of these complexities and

96 uncertainties, detection of an orbital signal in the Martian PLDs using spacecraft

97 observations poses a considerable challenge [Perron and Huybers, 2009].

98 The problem of orbital signal detection has been considered almost since the

99 PLDs were first discovered. Given the lack of an absolute chronology, most efforts to

100 interpret the PLDs have focused on modeling or analyzing their stratigraphy. The first

101 study to consider in detail how different PLD formation mechanisms influence the

102 resulting stratigraphy was that of Cutts and Lewis [1982]. They considered two

103 deposition models. In their first model, material composing the major constituent of the

104 PLDs is deposited at a constant rate, and differences between layers are caused by a

105 minor constituent that is deposited at a constant rate only when the obliquity of the planet

106 is below a certain threshold value. In their other model, only one type of material is 
107 deposited, but only when the obliquity is below a certain threshold value; layer

108 boundaries correspond to periods with no deposition. Although these models are highly

109 simplified, their work revealed the sensitivity of PLD stratigraphy to factors such as ice

110 deposition rates and thresholds, and thus hinted at the difficulty of detecting an orbital

111 signal. More recently, Levrard et al. [2007] used a global climate model for Mars to study

112 ice accumulation rates and concluded that formation of PLD layers must indeed be more

113 complex than originally modeled.

114 Other authors have used time series analysis to search for coherent signals in the

115 PLD stratigraphy, particularly signals that may be related to orbital forcing. Milkovich

116 and Head [2005] analyzed spectra of brightness profiles through the north PLDs, and

117 reported the presence of a signal with a $30 \mathrm{~m}$ vertical wavelength in the upper $300 \mathrm{~m}$ of

118 the PLDs, which they interpreted as a signature of the approximately $51 \mathrm{kyr}$ cycle of the

119 climatic precession. They assumed a linear time-depth relationship, however, and did not

120 evaluate the statistical significance of the signal they identified. Perron and Huybers

121 [2009] expanded this analysis, also assuming a linear time-depth relationship on average,

122 but allowing for local variability ("jitter") in this relationship. They also evaluated the

123 significance of peaks in the PLD spectra with respect to a noise background. Perron and

124 Huybers [2009] found that the PLD spectra closely resemble spectra for autocorrelated

125 random noise, but that many stratigraphic sequences contain intermittent, quasi-periodic

126 bedding with a vertical wavelength of $1.6 \mathrm{~m}$. Subsequent studies have confirmed and

127 refined this measurement of 1.6 meter bedding through analyses of higher-resolution

128 imagery and stereo topography [Fishbaugh, 2010; Limaye et al., 2012]. 
130 signals within the stratigraphy, but have not been able to conclusively identify evidence

131 of orbital forcing due to the absence of multiple periodic signals with a ratio of

132 wavelengths that matches the expected ratio of orbital periods [Perron and Huybers,

133 2009]. They have also been limited by the assumption of a linear time-depth relationship,

134 a scenario that, while possible, is rare in terrestrial stratigraphic sequences [Sadler, 1981;

135 Weedon, 2005]. Thus, while the Mars polar caps do appear to record repeating regional

136 or global climate events, the duration of these events and their relationship to orbitally

137 forced variations in insolation remain unknown.

138 In studies of terrestrial paleoclimate records, it is common to address the problem

139 of unknown time-depth relationships by tuning an observed record - adjusting its time

140 model nonuniformly by moving points in the record closer together or further apart - to

141 match an assumed forcing with a known chronology, or by tuning two or more observed

142 records with unknown chronologies to match each other. There have been limited efforts

143 to apply tuning procedures to the Mars PLDs. Laskar et al. [2002] compared the PLD

144 stratigraphy with an insolation time series using an approach in which a portion of the

145 photometric brightness image was stretched to provide an approximate fit to the

146 insolation time series. They analyzed only one image, however, and did not evaluate the

147 goodness of fit statistically. Milkovich et al. [2008] used the signal-matching algorithm of

148 Lisiecki and Lisiecki [2002] to search for stratigraphic correlations between PLDs in

149 different regions of the north polar cap, but did not attempt to tune PLD sequences to

150 match insolation records. 
The need to assess the statistical significance of proposed tunings is widespread in

152 the study of terrestrial paleoclimate [Proistosescu et al., 2012] and in other analyses that

153 seek correlations among time series with uncertain chronologies. The essential problem is

154 that any effort to tune records to match one another will produce some agreement, but it

155 is not clear whether this agreement arose by chance, or whether it reveals an underlying

156 relationship. To address this need, methods have been proposed that estimate the

157 significance of a tuned fit between records, generally by comparing the fit between

158 records that are hypothesized to share an underlying relationship with fits to random

159 records that share no underlying relationship with the observed record. This was the

160 general approach adopted by Milkovich et al. [2008] in their effort to correlate PLD

161 stratigraphic sequences with one another.

162 In this paper, we adapt a statistical procedure for evaluating the significance of

163 orbital tuning that has been successfully applied to terrestrial paleoclimate records and

164 has been shown to be applicable to comparisons between any two time-uncertain series

165 [Haam and Huybers, 2010]. We use the procedure to compare two data series -

166 insolation as a function of time and composition of strata as a function of depth - and

167 assess the potential for detecting an orbital signal in the Mars polar layered deposits. Our

168 approach is divided into two main steps. First, we construct simplified models for PLD

169 accumulation and drive these models with a Martian insolation time series to create

170 synthetic PLD records. We consider three different models, none of which produces a

171 linear time-depth relationship. In the second step, we perform a statistical analysis to

172 determine how reliably we can detect the orbital signal in the synthetic PLD records. The

173 statistical analysis uses a dynamic time warping algorithm to tune the synthetic PLD 
174 records to the insolation time series and a Monte Carlo procedure that evaluates the

175 statistical significance of that tuning by applying the same dynamic time warping

176 algorithm to random signals. For each modeled PLD formation mechanism, this

177 procedure yields an estimated confidence level for detection of an orbital signal. We then

178 consider the implications of this analysis for the interpretation of the PLD stratigraphic

179 sequences measured from spacecraft observations, including the prospects for identifying

180 evidence of orbital forcing. The purpose of our work is not to definitively identify the

181 accumulation function controlling PLD formation, but to assess the performance of a

182 technique that can be used to analyze PLD records that do not have a linear depth-age

183 relationship.

185 2. Polar Layered Deposit Formation Models

$186 \quad 2.1$ Insolation forcing

187 In the models presented here, hypothetical ice and dust deposition rates expressed

188 as functions of insolation are integrated forward in time to produce synthetic PLD

189 stratigraphic sequences. Changes in the seasonality and global distribution of insolation

190 on Mars are controlled mainly by the planet's climatic precession, obliquity variations,

191 and eccentricity variations [Ward, 1973, 1974, 1992; Touma and Wisdom, 1993; Laskar

192 et al., 2004]. The climatic precession of Mars has a period of approximately $51 \mathrm{kyr}$. The

193 obliquity of Mars varies with an average period of $120 \mathrm{kyr}$ due to variation of the spin

194 axis and is modulated by a $1200 \mathrm{kyr}$ period due to variation of its orbital inclination

195 [Ward, 1973]. The eccentricity of Mars's orbit varies with periods of $95 \mathrm{kyr}, 99 \mathrm{kyr}$ and

1962400 kyr [Laskar et al., 2004]. 
The evolution of Martian orbital parameters over long time intervals is chaotic

198 [Laskar and Robutel, 1993; Touma and Wisdom, 1993]. Given the precision with which

199 present-day orbital parameters can be measured, the current solution for insolation over

200 time [Laskar et al., 2004] is accurate for the last 10-20 Myr. We calculate insolation over

201 this interval from the orbital solution of Laskar et al. [2004] using methods described by

202 Berger [1978]. Like previous analyses of the PLDs [Laskar et al., 2002], we use the

203 average daily insolation at the north pole on the summer solstice (Fig. 2) as a proxy for

204 the climatic conditions controlling the deposition of polar ice and dust. This assumes that

205 the effect of the axial precession on the magnitude of ice deposition in a given year is less

206 important than the effect of obliquity. As noted above, our objective in this study is to

207 evaluate a procedure for analyzing PLD sequences with nonlinear time-depth

208 relationships, not to identify the exact relationship between insolation and PLD

209 formation, so our results do not rely on the correctness of this assumption.

210 The orbital solution features a significant reduction in mean obliquity, and

211 therefore summer insolation at the poles, after approximately 5 Ma. Paleoclimate models

212 suggest that polar ice caps would not have been stable before this time [Jakosky et al.,

213 1995; Mischna et al., 2003; Forget et al., 2006; Levrard et al., 2007], which would imply

214 that the PLDs exposed in the upper portions of the ice caps are younger than $5 \mathrm{Ma}$.

215 However, other studies have estimated the age of the southern PLDs to be an order of

216 magnitude older than this, which may be related to protective lag deposits [Banks et al.,

217 2010]. There is an observational constraint from crater counts that yields a maximum age

218 of $\sim 1 \mathrm{Ga}$ on the north polar basal units [Tanaka et al., 2008], and our approach does not 
219 depend on an estimate of the absolute age of the PLDs. In the models presented here, we

220 only consider the past five million years of Martian insolation history (Fig. 2).

221

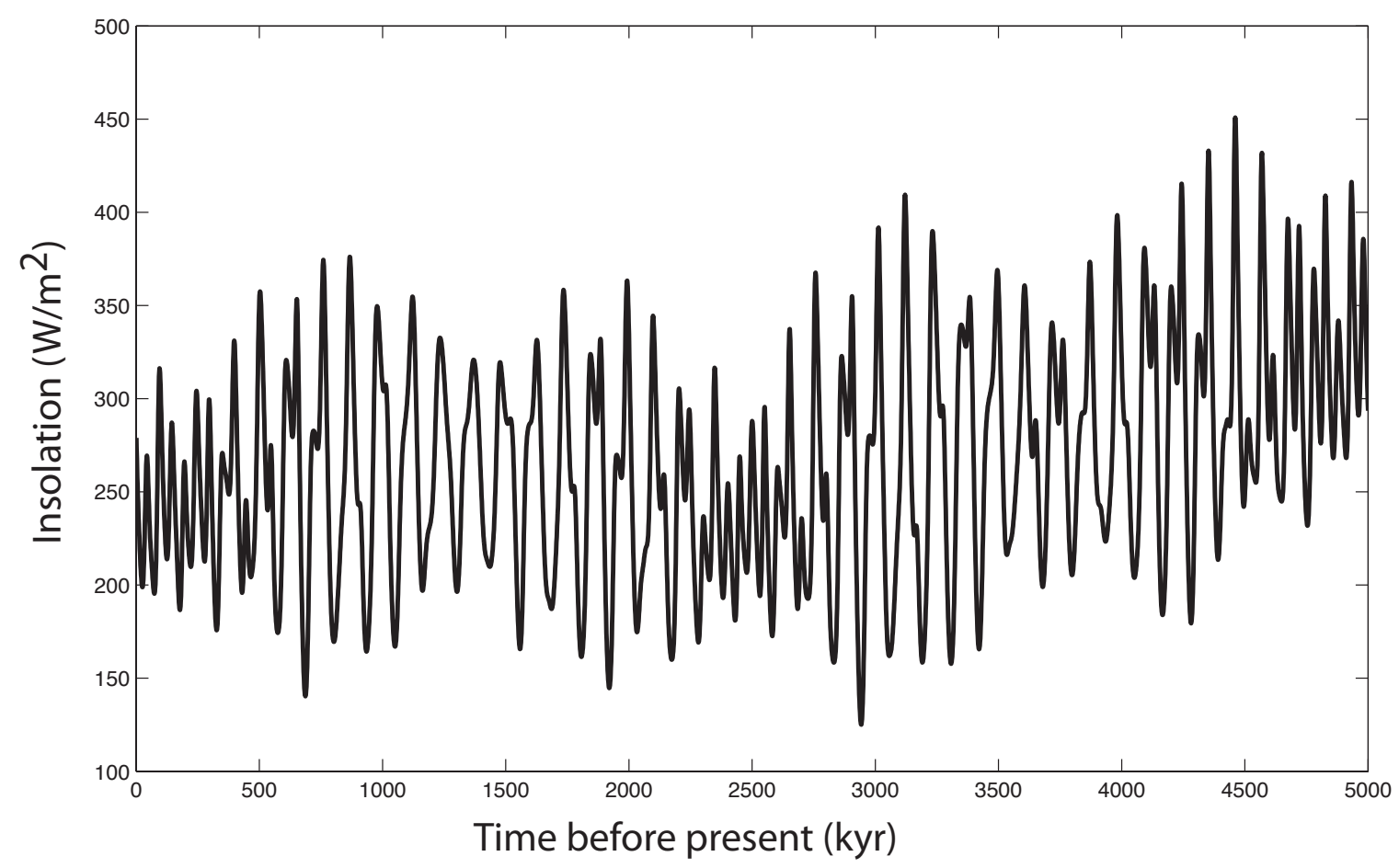

Figure 2. Martian insolation over the past five million years at the north pole on the summer solstice, calculated from the orbital solution of Laskar et al. [2004].

\subsection{Ice and dust accumulation}

225 We consider three classes of PLD formation models, which are illustrated

226 schematically in Figure 3. Although our models are more complicated than those

227 originally studied by Cutts and Lewis [1982], they are not intended to capture all aspects

228 of the physical processes controlling ice and dust deposition rates. The key attribute of

229 our simple, insolation-driven models is that they produce strata with a non-linear time-

230 depth relationship, and therefore provide a useful tool for exploring how insolation 
231 forcing may be recorded in the PLDs. In each model, dust deposition rate $f_{\text {dust }}[\mathrm{L} / \mathrm{T}]$ is

232 held constant, and ice deposition rate $f_{\text {ice }}[\mathrm{L} / \mathrm{T}]$ is expressed as a simple function of

233 insolation, $\phi\left(\mathrm{W} / \mathrm{m}^{2}\right)$. In the first model, ice deposition rate $f_{\text {ice }}(\phi)$ varies

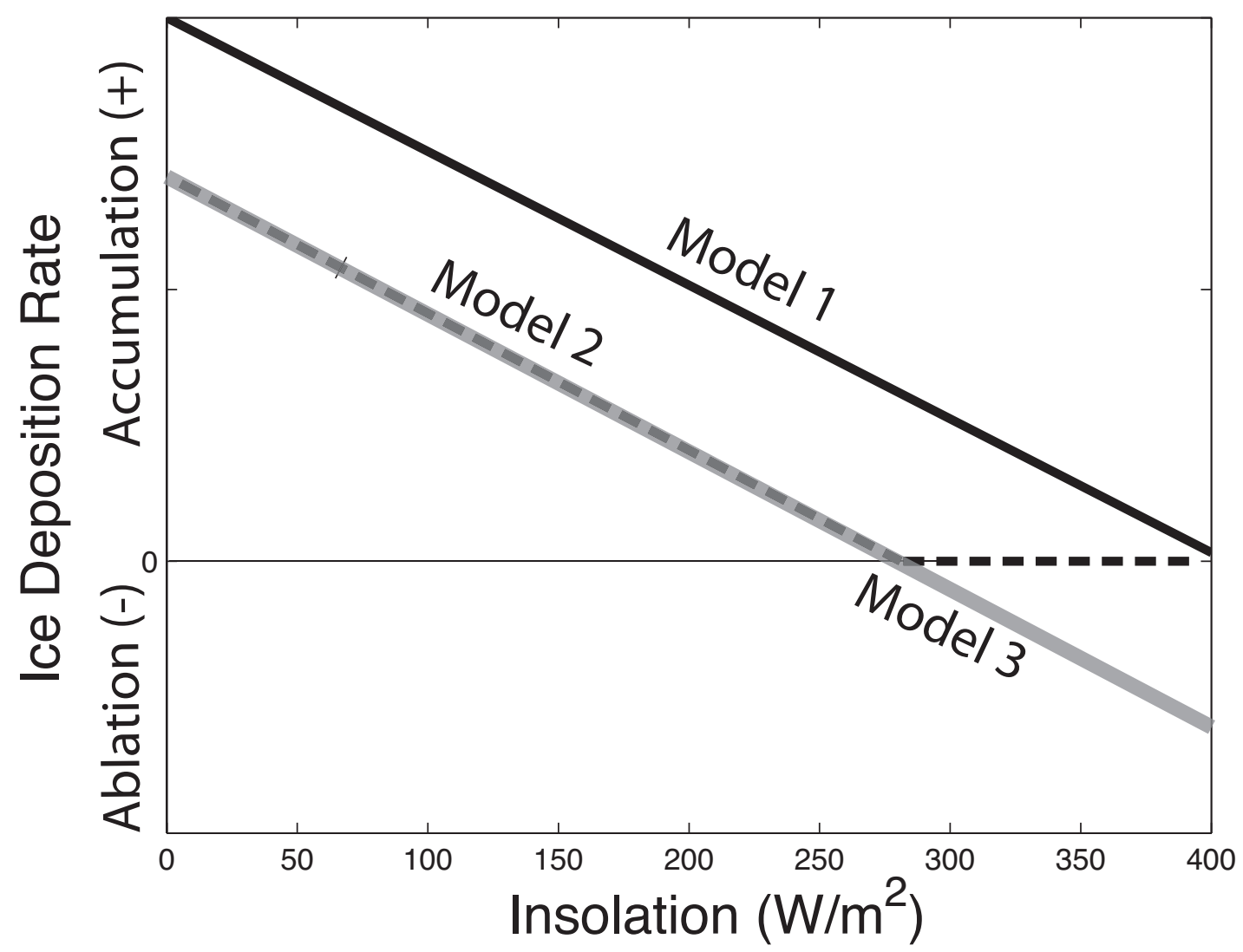

Figure 3. Ice deposition rate (arbitrary units) as a function of insolation for the three models considered. Model 1 (solid black line) is a simple linear dependence of deposition rate on insolation, with no hiatuses in deposition. Model 2 (dotted line) allows ice deposition rate to drop to zero at high insolation values, creating hiatuses. Model 3 (solid gray line) allows ice deposition rate to become negative at high insolation values, causing alabation of existing layers.

235 linearly with insolation. Higher insolation corresponds to slower ice deposition. The

236 insolation value at which no ice is deposited $\left(f_{\text {ice }}(\phi)=0\right)$ is chosen to be greater than the

237 maximum insolation reached in the past five million years, so that $f_{\text {ice }}(\phi)$ is always

238 positive, and the resulting PLDs contain no hiatuses in accumulation. 
240 which $f_{\text {ice }}(\phi)=0$ is chosen to be less than the maximum insolation reached in the past five

241 million years. For insolation values above this threshold, $f_{\text {ice }}(\phi)=0$. Therefore, for

242 certain time intervals in the past five million years, no ice is deposited, and the resulting

243 PLDs contain hiatuses in accumulation.

244 The third model is the same as the second model, except that $f_{\text {ice }}(\phi)$ maintains its

245 linear relationship with insolation at all insolation values, which means that $f_{\text {ice }}(\phi)$ is

246 negative for insolation values above the threshold. A negative ice deposition rate

247 corresponds to ablation, which destroys a previously deposited section of the PLD. The

248 resulting PLDs therefore contain hiatuses, as in the second model, but the hiatuses are not

249 limited to time intervals when insolation exceeds a threshold value. Figure 3 summarizes

250 the ice deposition functions for the three models. All three models can have their

251 parameters adjusted in order to vary the absolute values of their deposition rates. The

252 units of brightness and depth in the models are arbitrary, so the slopes of the trends

253 relating deposition rate to insolation in Figure 3 do not affect our tuning procedure.

\subsection{Generation of synthetic stratigraphic sequences}

256 For each instance of a model, the insolation time series (Fig. 2) is sampled every

2571000 years, for a total of 5000 time steps. At every time step, ice and dust deposition

258 rates are calculated, an increment of ice is deposited using a forward Euler method, and

259 the dust concentration of the ice is calculated as the ratio of the dust and ice deposition

260 rates. This iterative procedure constructs a synthetic PLD stratigraphic sequence 
261 consisting of a series of "beds" of unequal thickness and variable dust concentration.

262 Figure 4 shows examples of outputs for each model class.

263 The models make a number of simplifications. Dust is assumed to be

264 volumetrically negligible, on the basis of work that suggests an upper limit for dust

265 content of $2 \%$ by volume for the northern polar cap [Picardi et al., 2005]. Dust is

266 assumed to blow away during hiatuses in ice deposition, such that dust lags do not

267 develop in models with hiatuses or ablation. This assumption is consistent with abundant

268 evidence for eolian sediment transport in the north polar region [Byrne, 2009]. We

269 neglect topographic differences involving aspect and shadowing that could potentially

270 cause local variations in deposition rates, based on the observation that large-scale 


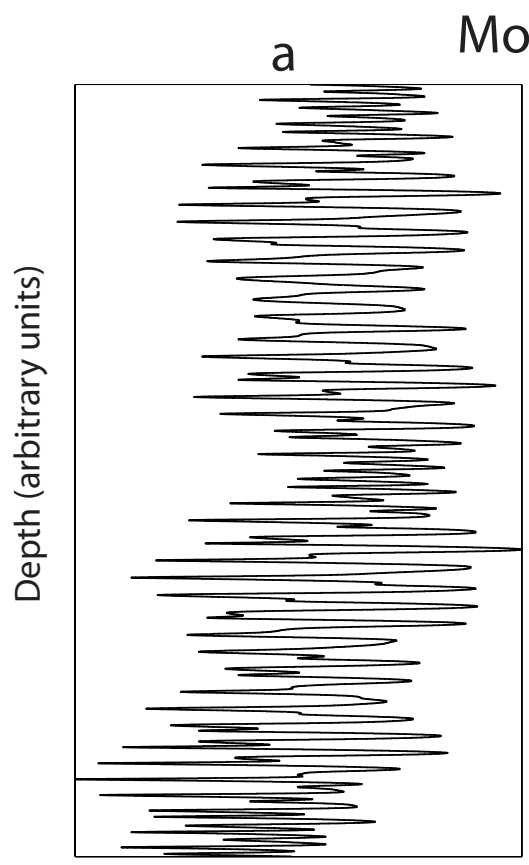

Brightness (arbitrary units)

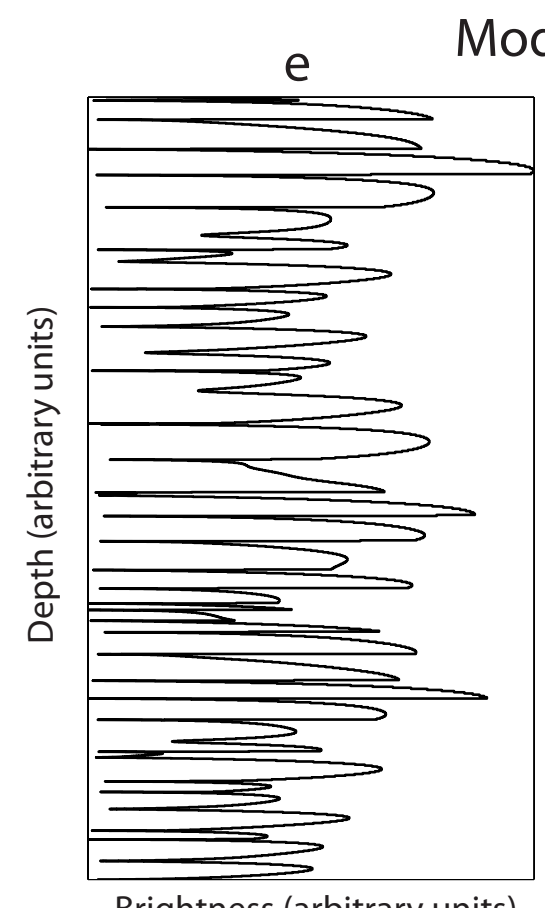

Brightness (arbitrary units)

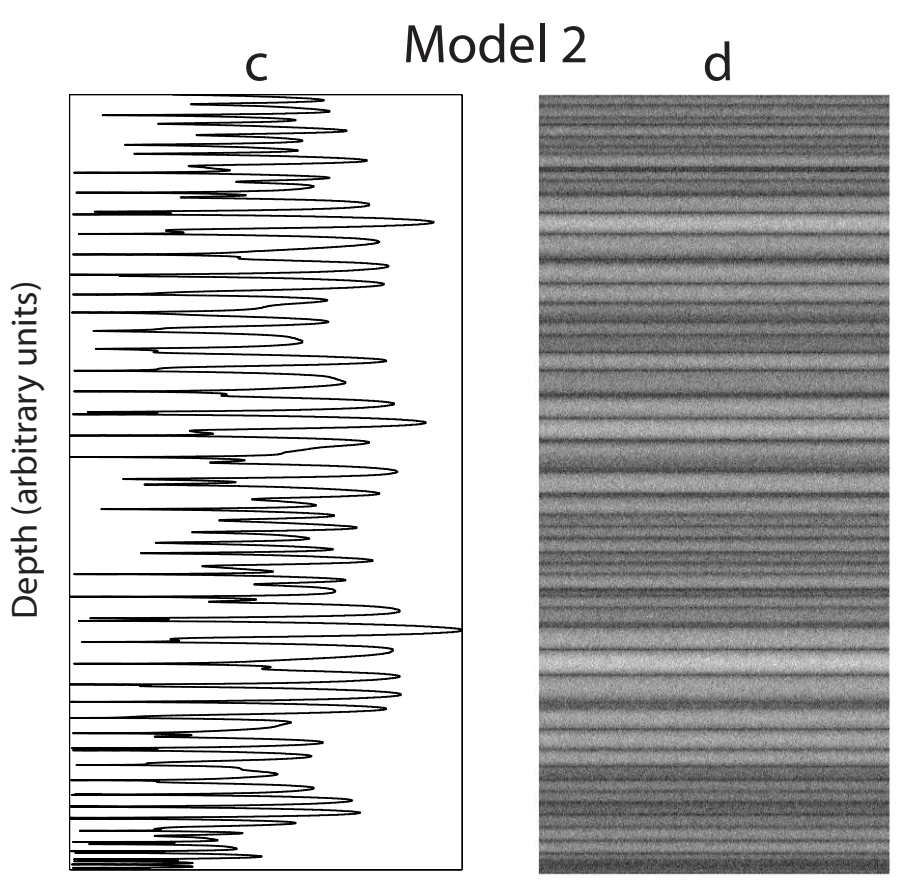

Brightness (arbitrary units)

Model 2, thresh. $200 \mathrm{~W} / \mathrm{m}^{2}$

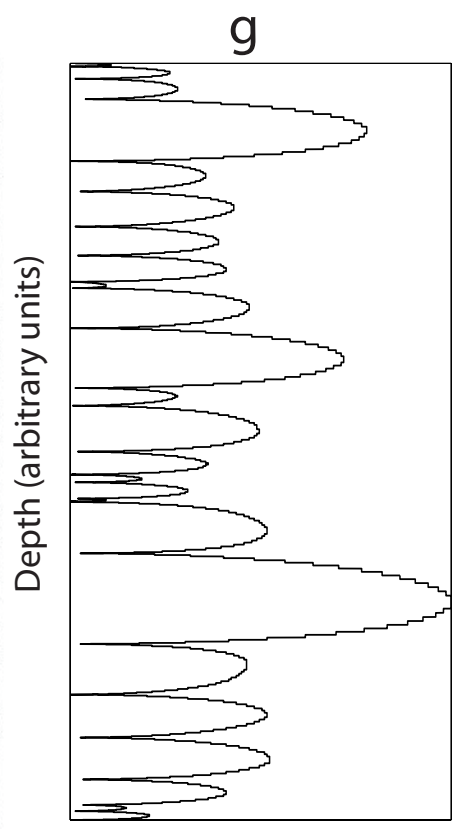

Brightness (arbitrary units)
Model 2, thresh. $250 \mathrm{~W} / \mathrm{m}^{2}$

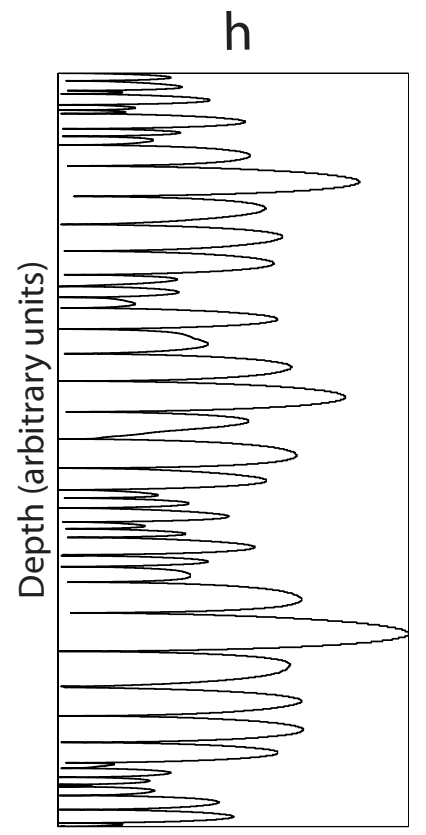

Brightness (arbitrary units)

Figure 4. Examples of synthetic PLD stratigraphic sequences produced by the three model classes. Plots in (a,c,e) show dust concentration in arbitrary units as a function of depth in arbitrary units. Images in (b,d,f) show simulated images of the stratigraphy (compare with Fig. 1) created by assuming that brightness scales inversely with dust concentration and adding Gaussian noise. The third model class (e,f), which includes ablation, produces synthetic PLDs most visually similar to actual images. Plots in $(\mathrm{g}, \mathrm{h})$ were both produced by the model with hiatuses and no ablation, but with different values of the threshold insolation for ice accumulation: $200 \mathrm{~W} / \mathrm{m}^{2}$ in $(\mathrm{g}), 250 \mathrm{~W} / \mathrm{m}^{2}$ in $(\mathrm{h})$. 
273 stratigraphy is consistent across the polar ice caps [Phillips et al., 2008]. In this study, we

274 have chosen to ignore insolation-induced variations in dust deposition rate, because we

275 expect ice deposition to be more strongly influenced by insolation [Toon et al., 1980].

276 Dust deposition rate is likely to be affected by global dust storms, which may correlate

277 with insolation [Zurek and Martin, 1993], but in the absence of a clear expectation for the

278 relationship between insolation and dust, and given the evidence that atmospheric

279 dustiness varies considerably over intervals much shorter than the periods of orbital

280 changes [Zurek and Martin, 1993], the relation between insolation and ice deposition rate

281 is a logical starting point. Stratigraphic thickness and dust concentrations are presented

282 in arbitrary units, because long-term deposition rates of ice and dust are poorly

283 constrained, with estimates spanning three orders of magnitude [Pollack et al., 1979;

284 Kieffer, 1990; Herkenhoff and Plaut, 2000]. This does not pose a problem for the tuning

285 procedure described below, because potential detection of an orbital signal involves

286 consideration of the relative amplitudes and frequencies of stratigraphic signals in PLD

287 records rather than the absolute dust concentrations and stratigraphic distances.

\section{3. Statistical Analysis}

290 Our statistical analysis consists of two main components: a dynamic time warping

291 algorithm that tunes a synthetic PLD record in an effort to match the insolation function,

292 and a Monte Carlo procedure that evaluates the statistical significance of the match. 
Revised for Icarus, November 2013

3.1 Orbital tuning by dynamic time warping

298 follow a linear time-depth relationship. We use a DTW algorithm proposed by Haam and

299 Huybers [2010] that tunes a record - stretches or contracts its time dimension

300 nonuniformly - to find the optimal match between the record and another time series.

301 The goodness of the match for a given tuning is measured by the covariance between the

302 tuned record and the other time series, and the optimal tuning is the one that maximizes

303 this covariance. In this case, the records are the synthetic PLDs, and they are tuned to

304 match the insolation function.

305 The DTW algorithm tunes the record to the forcing function by using a cost

306 matrix, which is constructed by computing the squared differences between each point in

307 the synthetic record and every point in the insolation function. The resulting matrix of

308 squared differences represents the costs (penalties) of all possible matches between points

309 in the two records. The algorithm then finds the path through the cost matrix that incurs

310 the lowest average cost, starting from an element that corresponds to the top of the PLD

311 record and the estimated time in the insolation function when the uppermost layer was

312 deposited, and ending at an element that corresponds to the bottom of the PLD record and

313 the time in the insolation function when the first layer was deposited. The calculated path

314 represents the tuned record that has the maximum possible covariance with the insolation

315 function. Figure 5 shows an example of an output of the DTW algorithm with both the

316 tuned and actual time-depth curves. The least-cost path is not required to terminate with

317 the earliest time in the insolation function; since most troughs only expose the uppermost 
318 few hundred meters of stratigraphy out of a total of $\sim 2 \mathrm{~km}$, it is likely that exposed

319 deposits only correspond to a fraction of the 5 Myr insolation function. Similarly, the

320 path is not required to start at the present day, because the uppermost strata may have

321 formed some time before the present. However, we expect that the age of the bottom of a

322 PLD sequence is much less certain than the age of the top, so we do not allow the starting

323 point of the least-cost path to vary as freely as the ending point. This is implemented by

324 imposing a non-zero cost on the leftmost column of the cost matrix and no cost on the

325 rightmost column (Fig. 5). We also impose a non-zero cost on the bottom row because a

326 path traveling along that row would correspond either to the unlikely scenario of a thick

327 layer of ice deposited instantaneously at the present day or to the unphysical scenario of

328 strata that are younger than the present. 


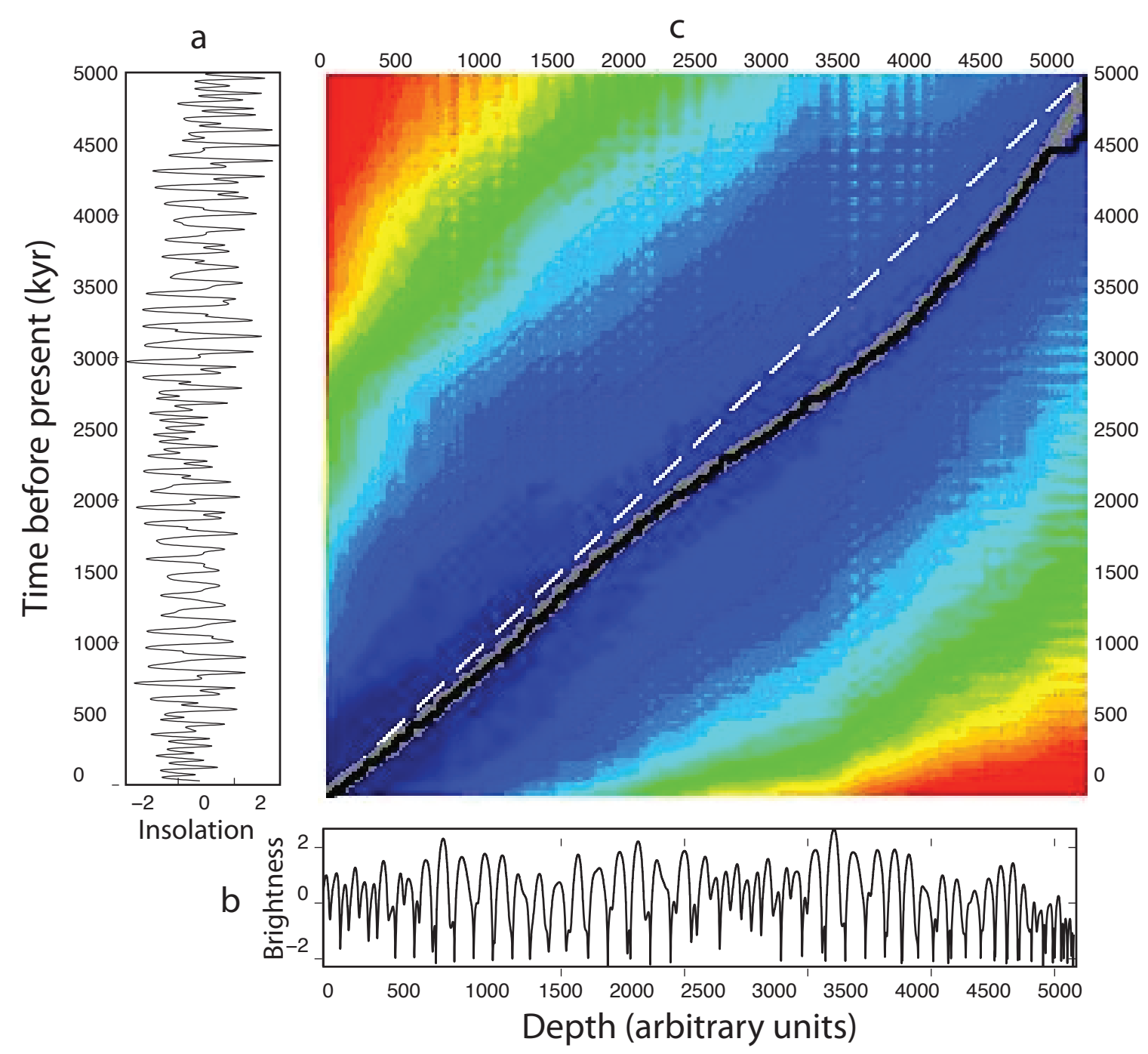

Figure 5. Output from the dynamic time warping algorithm comparing (a) the last five million years of Martian insolation history to (b) a synthetic PLD sequence. Both time series are normalized to unit variance. In the model, ice deposition stops (but without ablation) above a threshold insolation of $350 \mathrm{~W} / \mathrm{m}^{2}$. The square region (c) corresponds to the cost matrix. The black line in (c) shows the path through the cost matrix that incurs the lowest average cost, and represents the tuned synthetic PLD. The colors represent cost, with warm colors indicating areas of higher cost and cool colors indicating areas of lower cost. The dashed line in (c) is simply the diagonal of the cost matrix, which represents a linear time-depth relationship. The gray line in (c) represents the true time-depth relationship for this synthetic PLD. The covariance for this tuning is 0.963 despite the hiatuses in deposition. 
333 PLD and the insolation time series, but does not assign a statistical significance to that

334 covariance. The procedure therefore requires an additional step that quantitatively

335 evaluates the null hypothesis that the PLD record is a random time series uncorrelated

336 with insolation, and that the maximum covariance between the PLD and insolation is no

337 better than that obtained by chance. We evaluate this null hypothesis through a Monte

338 Carlo procedure in which random records with statistical characteristics similar to those

339 of the synthetic PLDs are tuned to match the insolation function. For each synthetic

340 PLD, 1000 random records with the same mean, variance, and lag-1 autocorrelation as

341 the synthetic PLD are generated. The DTW procedure then tunes each random record to

342 the insolation record using the same procedure applied to the synthetic PLD, yielding a

343 maximum covariance for each random record. A comparison of the resulting distribution

344 of 1000 maximum covariances with the maximum covariance between the insolation and

345 synthetic PLD provides a way of gauging the likelihood that the match is not spurious,

346 and therefore the confidence level at which the null hypothesis can be rejected. An

347 example is shown in Figure 6. We express this confidence level as the percentage of

348 random Monte Carlo records, $\mathrm{P}_{\mathrm{MC}}$, that yield a smaller maximum covariance than the

349 synthetic record. If $\mathrm{P}_{\mathrm{MC}}=100 \%$, then the synthetic PLD matches insolation better than

350 all random records, and the orbital signal is detected in the synthetic PLD with an

351 extremely high degree of confidence. If $\mathrm{P}_{\mathrm{MC}}=50 \%$, then the orbital signal is so obscured

352 by the PLD formation mechanism that the tuned match between the PLD and insolation is

353 no better than the median match between a random time series and insolation, and thus

354 there is little confidence that the modeled stratigraphy is related to insolation. Between 
355 these two extremes is a range of confidence levels for detection of an orbital signal. This

356 approach provides a way of quantifying the feasibility of detecting an orbital signal given

357 a hypothesized PLD formation mechanism, as well as a way of quantifying the

358 significance of orbital tuning applied to real PLD records, for which the formation

359 mechanism is unknown. Figure 7 compares dynamic time warping analyses of synthetic

360 PLD models and random time series for one case in which the covariance between

361 insolation and the tuned PLD is substantially higher than for the tuned random time series

362 (Fig. 7a,b) and another in which the PLD and random time series yield comparable

363 covariances (Fig. 7c,d).

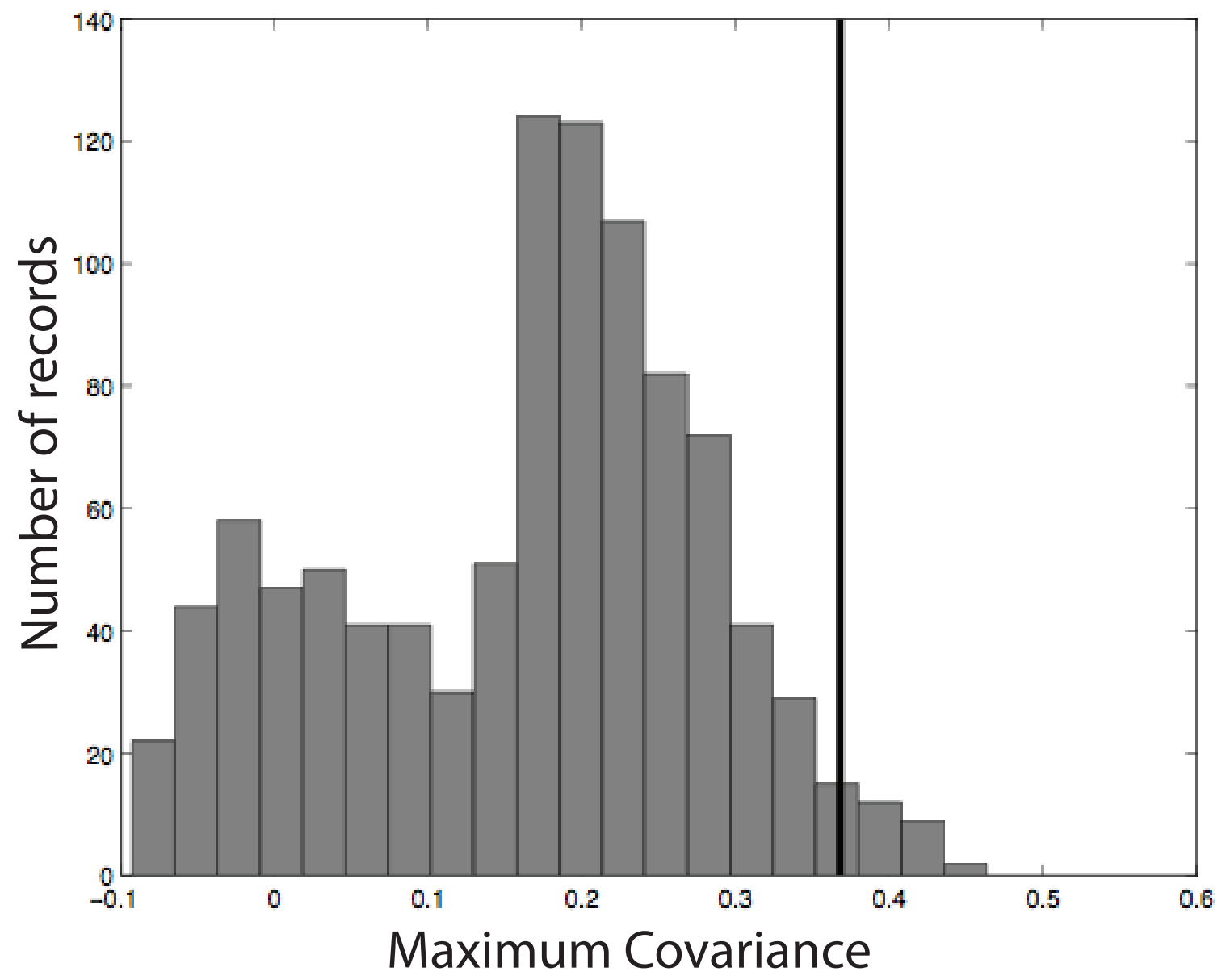

Figure 6. Histogram showing the distribution of maximum covariances for random records 
Revised for Icarus, November 2013

generated from a synthetic PLD where ablation occurs at a threshold insolation value of 270 $\mathrm{W} / \mathrm{m}^{2}$. The maximum covariance for the synthetic PLD tuned to the insolation record is 0.368 (shown here as the vertical black line), which is greater than $97.2 \%$ of the maximum covariances of the random records. We consider this a confident detection of the orbital signal. 


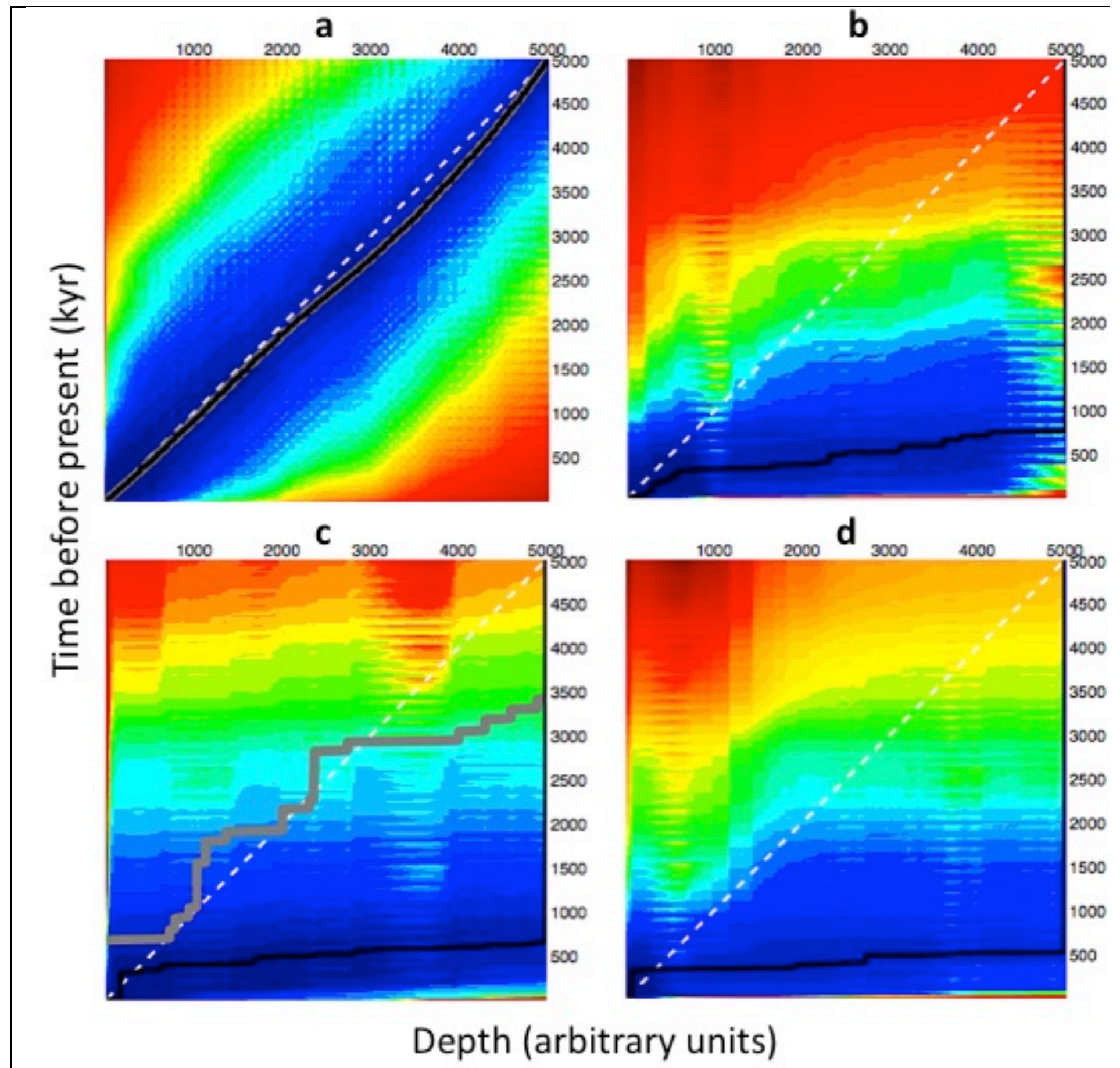

Figure 7. Cost matrices, as shown in Fig. 5, for four different dynamic time warping analyses. Plot (a) shows a synthetic PLD formed over $5 \mathrm{Myr}$ where ablation occurs at a threshold insolation value of $350 \mathrm{~W} / \mathrm{m}^{2}$ tuned to a $5 \mathrm{Myr}$ insolation signal. Plot (b) shows a corresponding random PLD tuned to the same signal. Plot (c) shows a synthetic PLD formed ove: Myr where ablation occurs at a threshold insolation value of $250 \mathrm{~W} / \mathrm{m}^{2}$ tuned to a $5 \mathrm{Myr}$ insolation signal. Plot (d) shows : corresponding random PLD tuned to the same signal. Note that the tuning in plot (a) is significantly better than that in plo (b), but there is no significant difference between plots (c) and (d). Solid black lines are the tuned time-depth relationships gray lines are the true time-depth relationships of the synthetic PLDs, and colors represent higher (warm colors) and lower (cool colors) costs, as in Fig. 5. 


\section{4. Results}

3684.1 Qualitative characteristics of synthetic PLD stratigraphy

369 Model outputs of synthetic PLD records yield noteworthy trends, even before 370 application of the DTW algorithm and Monte Carlo procedure. In the no-hiatus case,

371 where ice deposition rate varies linearly with insolation and is always positive (Fig. 4a,b),

372 varying the coefficient relating ice deposition rate to insolation changes the absolute

373 values of dust concentration in the resultant stratigraphic sequences, but not the relative

374 frequencies of bedding. The outcome of this simple formation model is therefore 375 qualitatively independent of model parameters.

376 The relative frequencies of bedding in models that allow hiatuses are also

377 insensitive to changes in the coefficient relating ice deposition rate to insolation (Fig. 4c-

378 f). However, adjusting the threshold insolation value in these models does change the

379 stratigraphy qualitatively, because it influences the fraction of time that is preserved.

380 Figure $4 \mathrm{~g}$,h shows two instances of the model with hiatuses but no ablation, with

381 different thresholds for ice deposition. Note that adjustment of this threshold changes not

382 only the values of dust concentration, but the number of bright peaks as well.

384 4.2 Detection of orbital signals for different accumulation models

385 As mentioned in section 3, a maximum covariance was calculated for each

386 synthetic PLD and was then compared to the maximum covariances obtained for 1000

387 randomly generated records that shared several statistical properties with the synthetic 
388 PLD. For models with no ablation and no hiatuses (Fig. 4a,b), the maximum covariance

389 is close to 1 and is always greater than the maximum covariances for all randomly

390 generated records $\left(\mathrm{P}_{\mathrm{MC}}=100 \%\right)$. Thus, for this simple formation function, we can

391 confidently identify an orbital signal in all cases, despite a nonlinear time-depth

392 relationship that would complicate or preclude detection with conventional time series

393 analysis methods. This result illustrates one of the main benefits of the tuning procedure,

394 and suggests that tuning analyses of the PLDs, combined with an appropriate statistical

395 test, could reveal underlying structure that conventional time series analyses have missed.

396 For the more complicated models that produce hiatuses (Fig. 4c-f), $\mathrm{P}_{\mathrm{MC}}$ generally

397 scales with the insolation threshold for ice deposition (Fig. 8a), because higher thresholds

398 result in shorter hiatuses. That is, when less of the insolation time series produces strata

399 that are preserved, the match between the PLDs and insolation is worse, and is less likely

400 to be better than the match to a random record. For sufficiently high insolation thresholds

$401\left(>225 \mathrm{~W} / \mathrm{m}^{2}\right.$ for the model with hiatuses but no ablation, and $>270 \mathrm{~W} / \mathrm{m}^{2}$ for the model

402 with ablation), the maximum covariance for the model output is greater than all

403 maximum covariances for random records $\left(\mathrm{P}_{\mathrm{MC}}=100 \%\right)$, despite incomplete

404 preservation of the modeled time interval (Fig. 8a). Below those threshold insolation

405 values, $\mathrm{P}_{\mathrm{MC}}$ decreases as the threshold is lowered. For models without ablation but with

406 ice deposition stopping above a threshold insolation value of $222 \mathrm{~W} / \mathrm{m}^{2}$, an orbital signal

407 can be detected with a 95\% degree of confidence. For a threshold insolation value of 174

$408 \mathrm{~W} / \mathrm{m}^{2}$ or lower, $\mathrm{P}_{\mathrm{MC}}$ is not significantly higher than $50 \%$, and thus the model output can

409 not be tuned to an orbital signal better than a random record; detection of an orbital signal

410 is infeasible. For models with ablation above a threshold insolation value of $269 \mathrm{~W} / \mathrm{m}^{2}$, 
411 an orbital signal can be detected with a $95 \%$ degree of confidence. For a threshold

412 insolation value of $243 \mathrm{~W} / \mathrm{m}^{2}$ or lower, $\mathrm{P}_{\mathrm{MC}}$ is not significantly higher than $50 \%$, and thus

413 the model output cannot be tuned to an orbital signal better than a random record;

414 detection of the signal is infeasible. For a threshold value of $210 \mathrm{~W} / \mathrm{m}^{2}$ or lower, no PLD

415 record exists - it is all ablated away.

416 We find that this relationship can be generalized by plotting $\mathrm{P}_{\mathrm{MC}}$ as a function of

417 the fraction of time preserved in the stratigraphy (Fig. 8b). For the formation models

418 investigated here, the modeled PLDs can be distinguished from random time series ( $\mathrm{P}_{\mathrm{MC}}$

$419>50 \%$ ) even if only a few percent of the modeled time interval is preserved in the

420 stratigraphy, and can be confidently distinguished ( $\left.\mathrm{P}_{\mathrm{MC}}>90 \%\right)$ if approximately $8-10 \%$

421 of the time interval is preserved. Between these extremes, $\mathrm{P}_{\mathrm{MC}}$ increases approximately

422 linearly with the fraction of time preserved.

423 We also examined the influence of the total duration of PLD accumulation on the

424 ease of identifying an orbital signal. In addition to the insolation time series for the past 5

425 Myr (Fig. 2), we drove the model that allows ablation with the insolation for the past 3

426 Myr and the past $1 \mathrm{Myr}$, and performed the same statistical analysis on the model

427 outputs. The results in Fig. 7 demonstrate that, in addition to the dependence on

428 insolation threshold, $\mathrm{P}_{\mathrm{MC}}$ is higher when the total accumulation interval is longer:

429 depositing the PLDs over a longer period of time makes it easier to detect an orbital 430 influence. 


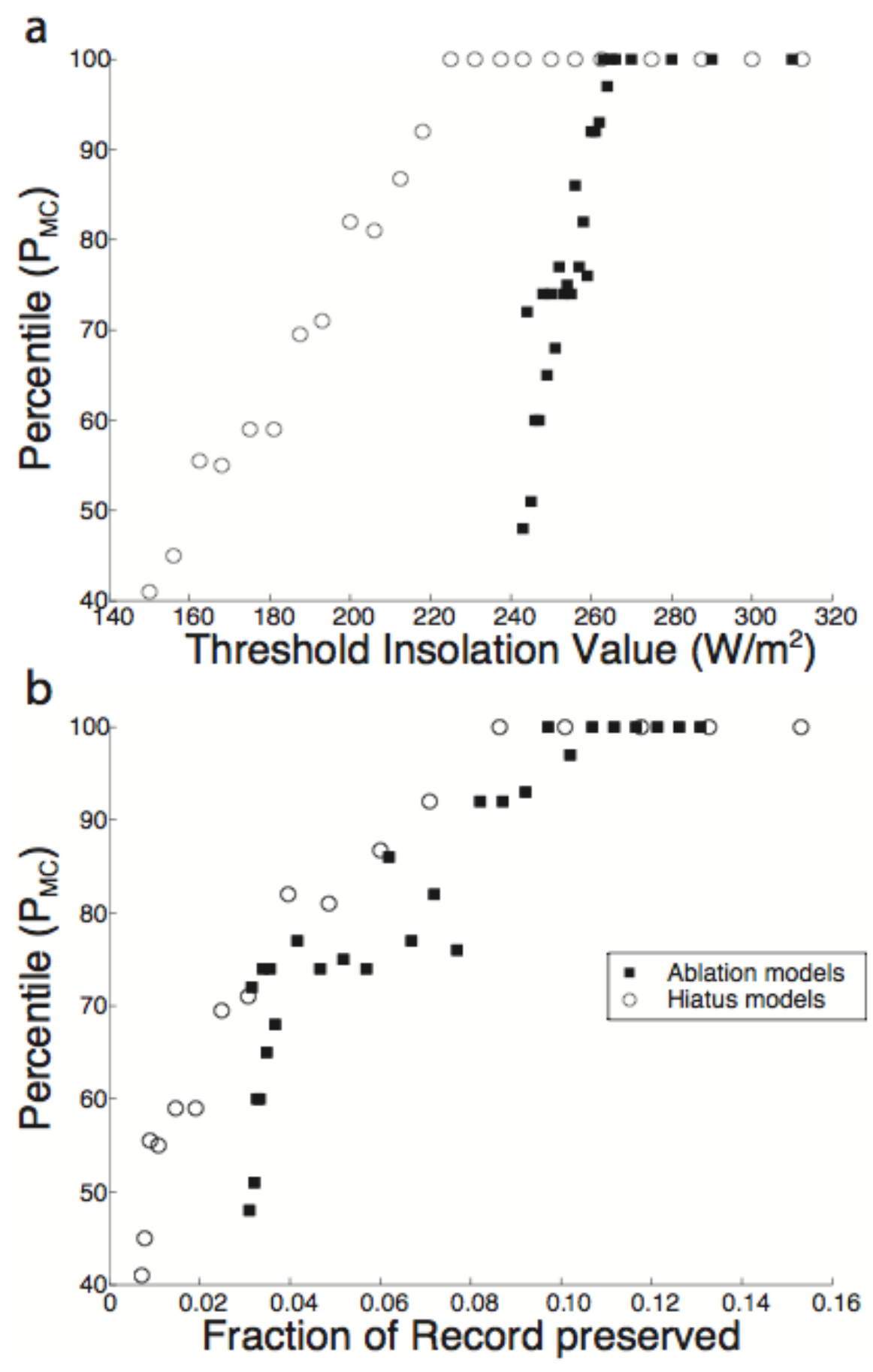

Figure 8. Percentage of randomly generated time series, $\mathrm{P}_{\mathrm{MC}}$, with insolation covariance that is smaller than the insolation covariance of a modeled PLD sequence, as a function of (a) the threshold insolation, and (b) the fraction of the $5 \mathrm{Myr}$ time interval that is preserved in the modeled stratigraphy. Trends for the model with hiatuses but no ablation and the model with hiatuses that do include ablation differ when $\mathrm{P}_{\mathrm{MC}}$ is compared with the magnitude of the insolation threshold for ice accumulation (a), but overlap when $\mathrm{P}_{\mathrm{MC}}$ is compared with the fraction of time preserved (b). 


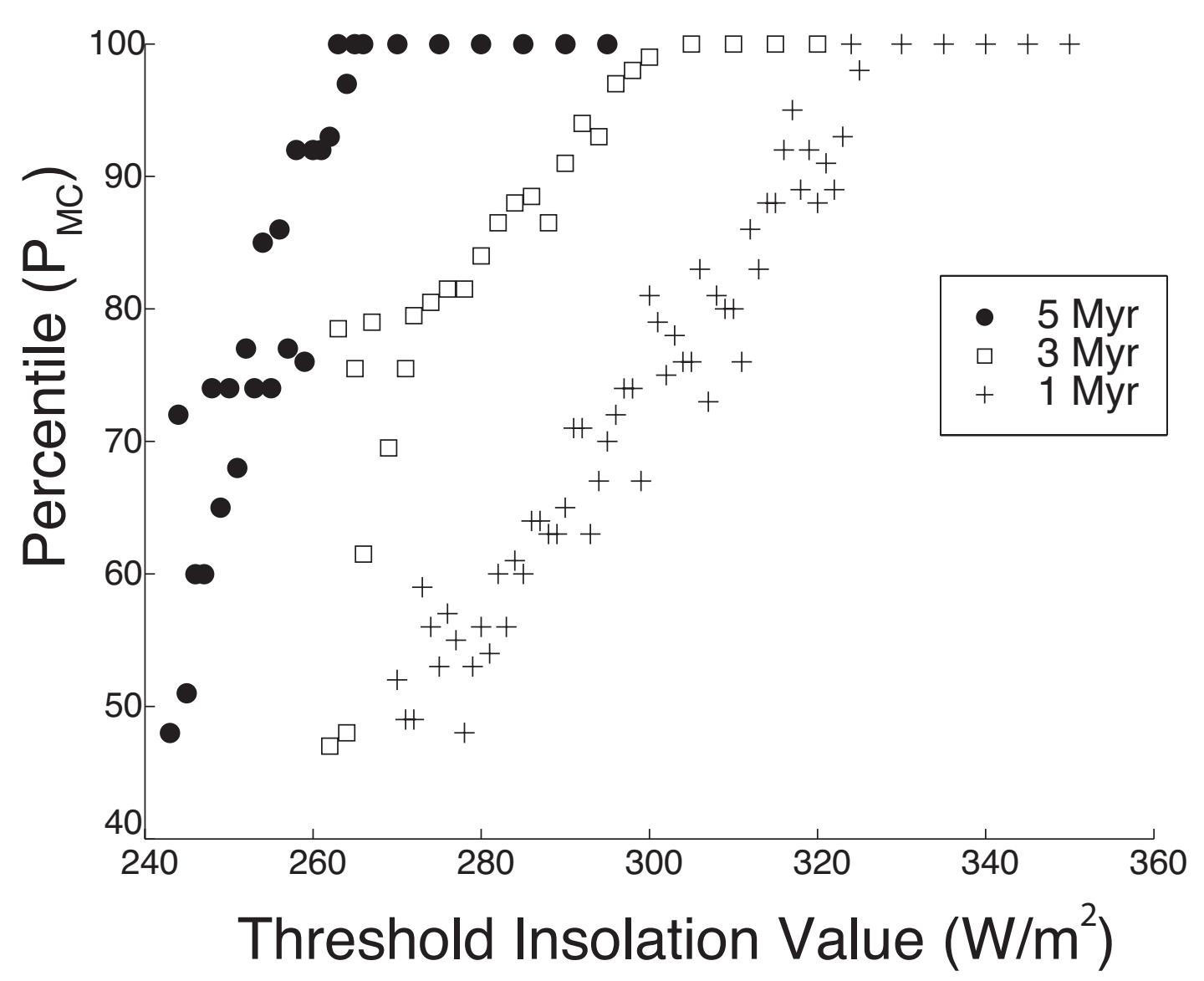

Figure 9. Percentage of randomly generated time series, $\mathrm{P}_{\mathrm{MC}}$, with insolation covariance that is smaller than the insolation covariance of a modeled PLD sequence, as a function of the insolation threshold for ice accumulation in the model that allows ablation. Different symbols correspond to models in which PLDs are deposited over the past 5, 3, or 1 million years of Martian history.

\section{5. Discussion}

$436 \quad 5.1$ Feasibility of identifying an orbital signal through tuning

437 In general, our results imply that detection of an orbital influence on PLD

438 formation is feasible (though not trivial), even if the relationship between depth and time

439 in the stratigraphy is strongly nonlinear. Indeed, we find that PLD sequences formed by

440 ice and dust deposition models that include no hiatuses in deposition can be distinguished

441 from stochastic time series $100 \%$ of the time. While such a deposition model is probably 
442 overly simple (see section 5.3), this result nonetheless emphasizes that a nonlinear time-

443 depth relationship is not an insurmountable complication.

444 In the more likely scenario that the PLD stratigraphy contains gaps, our analysis

445 provides a framework for determining whether the accumulated record contains enough

446 information to reliably identify orbital influence. Features such as unconformities and

447 crosscutting troughs suggest that the accumulation of the polar stratigraphic record was

448 punctuated by periods of no ice deposition [Tanaka et al., 2008]. In models with hiatuses

449 or ablation, the ability to detect orbital signals is a function of the threshold insolation at

450 which ice deposition stops. This result makes intuitive sense: when more of a PLD

451 record is ablated away, it is more difficult to detect the underlying forcing that drove PLD

452 formation. Our procedure identifies a clear, roughly linear relationship between the ease

453 of identifying an orbital influence, as measured by $\mathrm{P}_{\mathrm{MC}}$, and the insolation threshold for

454 ice deposition in each model (Fig. 8a). However, these particular values of the insolation

455 threshold should not be interpreted as absolute, because the true relationships between

456 insolation and ice and dust deposition rates are unknown. Instead, we emphasize that the

457 fraction of time preserved in the stratigraphy is the more relevant quantity for

458 determining whether an orbital signal can be confidently detected. The clearest

459 demonstration of this point is that the trends in $\mathrm{P}_{\mathrm{MC}}$ for the different models collapse to a

460 more uniform trend when plotted against fraction of time preserved (Fig. 8b) rather than

461 the threshold insolation (Fig. 8a).

462 The other main factor that influences the ease of detecting orbital influence is the 463 total duration of PLD formation. In general, the shorter the time period over which the 464 PLDs form, the more difficult it is to detect an orbital signal in the stratigraphy (Fig. 9). 
465 This too makes intuitive sense: a stratigraphic sequence that preserves $50 \%$ of five

466 million years contains more information than a sequence that preserves $50 \%$ of one

467 million years, and the additional information makes it easier to distinguish the orbitally

468 driven record from a random record.

$470 \quad 5.2$ Fraction of time preserved in the polar cap stratigraphy

471 Although the northern polar cap of Mars is thought to have experienced net

472 accumulation of ice over the past few Myr [Pollack, et al., 1979; Kieffer, 1990; Laskar et

473 al., 2002], it is unclear whether the cap is presently in a state of net accumulation or net

474 ablation. If we assume that Mars is in a state of net ablation today, then our models

475 suggest that the current PLDs represent only a small fraction $(<10 \%)$ of the total record

476 deposited over time. The current insolation at the Martian north pole during the summer

477 solstice, $265 \mathrm{~W} / \mathrm{m}^{2}$, is near the mean insolation for the past $5 \mathrm{Myr}$ of Martian history (Fig.

478 2). Thus, if the PLDs are ablating today, it is likely that they have ablated more often than

479 they have accumulated, and their strata may only record a small fraction of the past 5

480 Myr. It should be noted, however, that these models assume ablation occurs at a similar

481 rate to ice deposition. If ablation is much slower than ice deposition (which might be the

482 case if, for example, ablation forms a dust lag that inhibits further ablation), the PLDs

483 could record a larger portion of recent Martian history, even if the caps are experiencing

484 net ablation today.

485

486

487 


\subsection{Additional considerations for modeling PLD formation}

The objective of this study is to identify the main factors that influence the

490 viability of orbital tuning applied to the PLDs. We therefore have not attempted to

491 formulate a model for PLD accumulation that incorporates all the factors that influence

492 the appearance of the stratigraphy, nor have we attempted an absolute calibration of rate

493 parameters. Nonetheless, given the finding that orbital tuning may indeed be a viable

494 means of identifying the cause of paleoclimate signals preserved in the PLDs, it is

495 important to consider the limitations of, and possible improvements to, the simple models

496 presented here.

497 Several improvements could be implemented to make the PLD formation models

498 more realistic. In particular, both ice and dust deposition rates could be expressed in

499 terms of a fuller complement of physical variables. Ice deposition rates could take

500 humidity into account. Dust deposition rates could consider the occurrence of global dust

501 storms, which historical observations [Pollack et al., 1979; Toon et al., 1980; Haberle,

502 1896; Zurek and Martin, 1993] suggest produce a high frequency signal, but which may

503 also include long-term trends related to insolation [Fernandez, 1998]. These additional

504 complexities will almost certainly make detection of an orbital signal more difficult, and

505 thus the confidence in detection abilities presented in this study should be interpreted as

506 an upper limit.

507 Other potential complications are the possibility of stochastic variability in

508 deposition processes and the imperfect relationship between PLD composition and

509 appearance. To explore how these factors influence the orbital tuning procedure, we

510 performed an additional analysis in which the modeled ice deposition rate includes a 
511 stochastic component. Specifically, we added red noise (a random signal in which

512 spectral power $P$ declines with frequency $f$ according to $P \propto f^{-2}$ ) to the amount of ice

513 deposited in a given time step in our models to generate synthetic PLDs that are not

514 constructed with the assumption of a deterministic relationship between ice deposition

515 rate and insolation. Starting with a model that forms hiatuses when the insolation is 300

$516 \mathrm{~W} / \mathrm{m}^{2}$ or greater, we varied the amplitude of the noise and produced 100 random

517 realizations of the PLD strata for each value of noise amplitude. We then used the DTW

518 algorithm to calculate the maximum covariance between each modeled stratigraphic

519 sequence and the insolation time series. Figure 10 shows how the maximum covariance

520 depends on the amplitude of the noise. The addition of red noise to the ice deposition

521 rate changes the maximum covariance in a gradual fashion, suggesting that a non-

522 deterministic relationship between insolation and PLD accumulation does not necessarily

523 prevent the DTW method from identifying an orbital signal. 


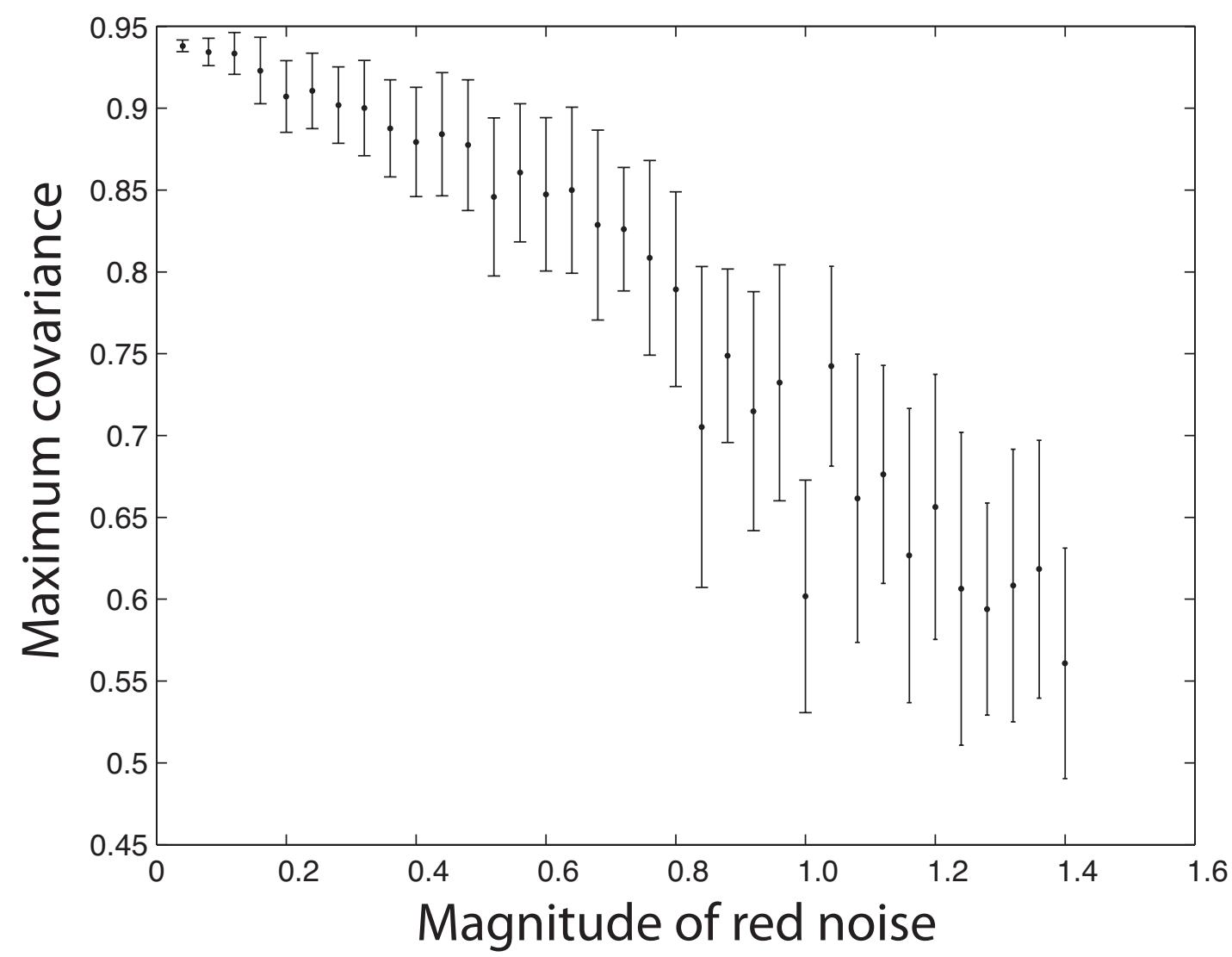

Figure 10. Effect of adding red noise to the modeled ice deposition rate on the dynamic time warping algorithm's ability to tune the resulting synthetic PLD to the insolation signal. The magnitude of the noise added is the ratio of the variance of the noise to the variance of the ice deposition rate. Each point represents the average maximum covariance of 100 different tunings of a synthetic PLD that contains hiatuses when the insolation reaches a value of $300 \mathrm{~W} / \mathrm{m}^{2}$ or greater. Error bars are one standard deviation.

\subsection{Implications for orbital tuning of the observed PLD stratigraphy}

527 Given the probable influence of insolation on the deposition or ablation of water

528 ice, the major constituent of the PLDs, it is likely that the relationship between time and

529 depth in the PLDs is nonlinear, as our simple models predict. One of the main

530 implications of our results is that it may nonetheless be possible to identify evidence of 531 quasi-periodic insolation forcing by applying a tuning procedure like the one described 
532 here. Such an analysis could reveal coherent signals in the PLD stratigraphy that would

533 not be detected by conventional time series analysis procedures that assume a linear or

534 nearly linear time-depth relationship [Perron and Huybers, 2009].

535 The appropriate future direction of this study is to apply the statistical analysis

536 described here to actual images of the Martian PLDs. Images obtained by the Mars

537 Orbiter Camera (MOC) on the Mars Global Surveyor spacecraft and the High Resolution

538 Imaging Science Experiment (HiRISE) aboard the Mars Reconnaissance Orbiter can be

539 converted to sequences of brightness vs. depth that can be analyzed with the same

540 procedure as the synthetic sequences of dust concentration studied here [Milkovich and

541 Head, 2005; Milkovich et al., 2008; Perron and Huybers, 2009; Fishbaugh, 2010; Limaye

542 et al., 2012]. These sequences can be compared to insolation records of varying lengths,

543 with the important consideration that a match would only determine the age of the

544 exposed sequence; such an age would be younger than that of the entire PLDs, which

545 would need to be extrapolated. It should be noted that conversion of images to

546 brightness-depth sequences introduces an additional source of noise that must be

547 considered [Tanaka, 2005], but recent efforts to quantify these uncertainties have found

548 them to be modest [Limaye et al., 2012]. The dynamic time warping procedure we have

549 applied to brightness records can in principle be applied to other proxies for PLD

550 composition, such as sequences of slope or roughness vs. depth, or composite records

551 incorporating both brightness and topographic information. Thus, for any possible

552 identification of an orbital signal in the PLDs, the statistical procedure presented here can

553 yield a quantitative estimate of the likelihood of a spurious match. If the PLDs preserve a

554 sizeable fraction of the total accumulation time, and the deposition rates of ice and dust 
555 are sufficiently deterministic, it may well be possible to detect an orbital signal, if one is

556 present.

\section{6. Conclusions}

559 We use a statistical procedure that evaluates the significance of time series tuning

560 to examine the feasibility of detecting an influence of orbital variations on the polar

561 stratigraphy of Mars. We apply the procedure to synthetic stratigraphic sequences

562 generated by simple formation models for the Martian polar layered deposits, and find

563 that detection of an orbital signal in the resulting stratigraphy is feasible, though not

564 trivial. Models in which ice deposition rate varies linearly with insolation produce

565 stratigraphy in which orbital signals are easily detected with the tuning procedure, despite

566 a nonlinear relationship between depth and time that can foil conventional time series

567 analysis methods. For more complicated models of ice deposition, detection ability

568 depends strongly on the threshold insolation at which ice deposition stops or an ablation

569 episode begins, and more generally, on the fraction of total formation time preserved in

570 the strata. Improved constraints on ice and dust deposition rates on Mars would permit a

571 more definitive assessment of whether detection of an orbital signal in the PLDs is

572 feasible, but our analysis does not reveal the problem to be necessarily intractable at the

573 current state of knowledge. HiRISE images should be adequate to identify evidence of

574 an orbital influence if PLD formation is controlled by a sufficiently simple mechanism,

575 but for certain formation scenarios described here, confident identification of an orbital

576 signal is impossible without absolute ages, no matter the quality of the spacecraft images. 
Revised for Icarus, November 2013

578

579

580

581

582

583

584

585

586

587

588

589

590

591

592

593

594

595

596

597

598

599

600

601

602

603

604

605

606

607

608

609

610

611

612

613

614

615

616

617

618

\section{Acknowledgments}

This study was supported by the NASA Mars Data Analysis Program, award 65P-

1089493.

\section{References}

Banks, M.E., et al., 2010. Crater population and resurfacing of the Martian north polar layered deposits. J. Geophys. Res. 115, E08006, doi:10.1029/2009JE003523.

Berger, A.L., 1978. Long-term variations of daily insolation and Quaternary climatic changes. J. Atmos. Sci. 35, 2362-2367

Byrne, S., 2009. The Polar Deposits of Mars. Annu. Rev. Earth Planet. Sci 37, 535-560.

Cutts, J.A., 1973. Nature and origin of layered deposits of the Martian polar region. J. Geophys. Res. 78, 4231-4249.

Cutts, J.A. et al., 1976. North Polar Region of Mars: Imaging results from Viking 2. Science 194, 1329-1337.

Cutts, J.A., and Lewis, B.H., 1982. Models of Climate Cycles Recorded in Martian Polar Layered Deposits. Icarus 50, 216-244.

Fernandez, W, 1998. Martian Dust Storms: A Review. Earth, Moon, and Planets 77, 19-46.

Fishbaugh, K.E., and Hvidberg, C.S., 2006. Martian north polar layered deposits stratigraphy: Implications for accumulation rates and flow. J. Geophys. Res. 111.

Fishbaugh, K.E., et al., 2010. First high-resolution stratigraphic column of the Martian north polar layered deposits. Geophys. Res. Letters 37.

Forget, F. et al., 2006. Formation of glaciers on Mars by atmospheric precipitation at high obliquity. Science 311, 368-371.

Haam, E., and Huybers, P., 2010. A test for the presence of covariance between timeuncertain series of data with application to the Dongge Cave speleothem and atmospheric radiocarbon records. Paleoceanography 25.

Haberle, R.M., 1986. Interannual variability of global dust storms on Mars. Science 234, 459-461.

Hays, J.D., Imbrie, J., and Shackleton, N.J., 1976. Variations in the Earth's orbit: Pacemaker of the Ice Ages. Science 194, 1121-1132.

Herkenhoff, K., and Plaut, J.J., 2000. Surface ages and resurfacing rates of the polar layered deposits on Mars. Icarus 144, 243-253.

Herkenhoof, K.E., et al., 2007. Meter-scale morphology of the north polar region of Mars. Science 317, 1711-1715.

Howard, A.D., Cutts, J.A., and Blasius, K.R., 1982. Stratigraphic relationships between Martian polar cap deposits. Icarus 50, 161-215.

Jakosky, B.M., Henderson, B.G., and Mellon, M.T., 1995. Chaotic obliquity and the nature of the Martian Climate. J. Geophys. Res. 100, 1579-1594.

Kieffer, H.H, 1990. $\mathrm{H}_{2} \mathrm{O}$ grain size and the amount of dust in Mars residual north polar cap. J. Geophys. Res. 95, 1481-1493. 
Kominz, M.A., and Pisias, N.G., 1979. Pleistocence climate: Deterministic or stochastic? Science 204, 171-173.

Koutnik, M., Byrne, S., and Murray, B., 2002. South Polar Layered Deposits of Mar: The cratering record. J. Geophys. Res., 107, 5100, doi:10.1029/2001JE001805.

Laskar, J. et al., 2004. Long term evolution and chaotic diffusion of the insolation quantities of Mars. Icarus 170: 343-64.

Laskar, J., Levrard, B., and Mustard, J.F., 2002. Orbital forcing of the Martian polar layered deposits. Nature 419, 375-377.

Laskar, J., and Robutel, P., 1993. The chaotic obliquity of the planets. Nature 361, 608-612.

Levrard, B., et al., 2007. Recent formation and evolution of northern Martian polar layered deposits as inferred from a Global Climate Model. J. Geophys. Res. 112.

Limaye, A.B.S., O. Aharonson and J.T. Perron. Detailed stratigraphy and bed thickness of the Mars north and south polar layered deposits. J. Geophys. Res., in review, 2012.

Lisiecki, L. E., and P. A. Lisiecki, 2002, Application of dynamic programming to the correlation of paleoclimate records. Paleoceanography, 17(D4), 1049.

Mellon, M.T., 1996. Limits on the $\mathrm{CO}_{2}$ content of the Martian polar layered deposits. Icarus 124, 268-279.

Milkovich S.M., and Head J.W. III, 2005. North polar cap of Mars: Polar layered deposit characterization and identification of a fundamental climate signal. J. Geophys. Res. 110, 1005

Milkovich, S.M., et al., 2008. Stratigraphic analysis of the northern polar layered deposits of Mars: implications for recent climate history. Planetary and Space Science 56(2), 266-288.

Mischna, M.A., et al., 2003. On the orbital forcing of Martian water and $\mathrm{CO}_{2}$ cycles: $\mathrm{A}$ general circulation model study with simplified volatile schemes. J. Geophys. Res. 108, 5062.

Murray, B.C. et al., 1972. Geological Framework of the South Polar Region of Mars. Icarus 17, 328-345.

Murray, B.C., Ward, W.R., and Yeung S.C., 1973. Periodic insolation variations on Mars, Science 180, 638-640.

Nye, J.F. et al., 2000. The instability of a south polar cap on Mars composed of carbon dioxide. Icarus 144, 449-455.

Perron, J.T., and Huybers, P, 2009. Is there an orbital signal in the polar layered deposits on Mars? Geology 37, 155-158.

Phillips, R.J. et al., 2008. Mars north polar deposits: Stratigraphy, age, and geodynamical response. Science 320, 1182-1185.

Pollack, J.B. et al., 1979. Properties and effects of dust particles suspended in the Martian atmosphere. J. Geophys. Res. 84, 2929-2945.

Plaut, J.J. et al., 2007. Subsurface radar sounding of the south polar layered deposits of Mars. Science 316, 92-96.

Picardi, G. et al., 2005. Radar soundings of the subsurface of Mars. Science 310, 1925-1928.

Proistosescu, C., P. Huybers and A.C. Maloof, 2012. To Tune or not to Tune: Detecting Orbital Variability in Oligo-Miocene Climate Records. Earth and Planetary 
665

666

667

668

669

670

671

672

673

674

675

676

677

678

679

680

681

682

683

684

685

686

687

688

689

690

691

692

693

694

695

696

697

698

699

700

Science Letters, 325-326, 100-107.

Sadler, P.M., 1981. Sediment accumulation rates and the completeness of stratigraphic sections, J. Geol. 89, 569-584.

Smith, D.E., Zuber, M.T., and Neumann, G.A., 2001. Seasonal variations of snow depth of Mars. Science 294, 2141-2145.

Tanaka, K.L., 2005. Geology and insolation-driven climatic history of Amazonian north polar materials on Mars. Nature 437, 991-994.

Tanaka, K.L. et al., 2008. North polar region of Mars: Advances in stratigraphy, structure, and erosional modification. Icarus 196, 318-358.

Thomas, P. et al., 1992. Polar deposits of Mars in Kieffer, H.H., et al., Mars. Tucson, University of Arizona press, 767-795.

Toon, O.B. et al., 1980. The astronomical theory of climatic change on Mars. Icarus 44, $552-607$.

Touma, J., and Wisdom, J., 1993. The chaotic obliquity of Mars. Science 259, 1294-1297.

Ward, W.R., 1973. Large-scale variations in the obliquity of Mars, Science 181, 260-262.

Ward, W. R., 1974, Climatic variations on Mars: 1. Astronomical theory of insolation. J. Geophys. Res., 79, $3375-3386$.

Ward, W. R., 1992, Long-term orbital and spin dynamics of Mars, in Mars, edited by H. H. Kieffer et al., chap. 9, pp. 298 - 320, Univ. of Ariz. Press, Tucson.

Weedon, G., 2003. Time-Series Analysis and Cyclostratigraphy. Cambridge, Cambridge University Press.

Wieczorek, M.A., 2008. Constraints on the composition of the martian south polar cap from gravity and topography. Icarus 196, 506-517.

Wunsch, C., 2004. Quantitative estimate of the Milankovitch-forced contribution to observed Quaternary climate change, Quaternary Sci. Rev. 23, 1001-1012.

Zurek, R., and Martin, L., 1993. Interannual variability of planet-encircling dust storms on Mars. J. Geophys. Res. 98, 3247-3259.

Zuber, M.T. et al., 1998. Observations of the north polar region of Mars from the Mars orbiter laser altimeter. Science 282, 2053-2060.

Zuber, M.T., et al., 2007. Density of Mars' south polar layered deposits. Science 300, 299-303. 